\title{
New Exact Solutions of Kolmogorov Petrovskii Piskunov Equation, Fitzhugh Nagumo Equation, and Newell- Whitehead Equation
}

\author{
Yu-Ming Chu $\mathbb{D}^{1,2}$ Shumaila Javeed $\mathbb{D}^{1},{ }^{3}$ Dumitru Baleanu, ${ }^{4,5}$ Sidra Riaz, ${ }^{6}$ \\ and Hadi Rezazadeh ${ }^{7}$ \\ ${ }^{1}$ Department of Mathematics, Huzhou University, Huzhou 313000, China \\ ${ }^{2}$ Hunan Provincial Key Laboratory of Mathematical Modeling and Analysis in Engineering, Changsha University of Science \\ \& Technology, Changsha 410114, China \\ ${ }^{3}$ Department of Mathematics, COMSATS University Islamabad, Park Road, Chak Shahzad, Islamabad, Pakistan \\ ${ }^{4}$ Department of Mathematics, Cankaya University, Ankara, Turkey \\ ${ }^{5}$ Institute of Space Sciences, Magurele-Bucharest, Romania \\ ${ }^{6}$ Department of Mathematics, Riphah International University, Sector I-14, Islamabad, Pakistan \\ ${ }^{7}$ Faculty of Engineering Technology, Amol University of Special Modern Technologies, Amol, Iran
}

Correspondence should be addressed to Shumaila Javeed; shumaila_javeed@comsats.edu.pk

Received 13 June 2020; Revised 28 September 2020; Accepted 8 October 2020; Published 9 November 2020

Academic Editor: Zengtao Chen

Copyright ( $2020 \mathrm{Yu}$-Ming Chu et al. This is an open access article distributed under the Creative Commons Attribution License, which permits unrestricted use, distribution, and reproduction in any medium, provided the original work is properly cited.

\begin{abstract}
This work presents the new exact solutions of nonlinear partial differential equations (PDEs). The solutions are acquired by using an effectual approach, the first integral method (FIM). The suggested technique is implemented to obtain the solutions of spacetime Kolmogorov Petrovskii Piskunov (KPP) equation and its derived equations, namely, Fitzhugh Nagumo (FHN) equation and Newell-Whitehead (NW) equation. The considered models are significant in biology. The KPP equation describes genetic model for spread of dominant gene through population. The FHN equation is imperative in the study of intercellular trigger waves. Similarly, the NW equation is applied for chemical reactions, Faraday instability, and Rayleigh-Benard convection. The proposed technique FIM can be applied to find the exact solutions of PDEs.
\end{abstract}

\section{Introduction}

The nonlinearity in the world prevails thoroughly; thus, it is significant to develop nonlinear models including partial differential equations [1-4]. Nonlinear conformable PDEs attracted the interest of many researchers because of their vast applications in different fields, for example, in chemistry, acoustic, fluid dynamics, image processing, biology, physics, vibration, and control [5-7]. Nonlinear conformable PDEs have a great potential to apply in several fields; thus, researchers put noteworthy attention for their analytical and numerical solutions [8-12]. Different effective and reliable techniques are proposed like the homotopy analysis method [13], homotopy perturbation technique [14], extended hyperbolic tangent method $[15,16]$, hyperbolic function method [17], subequation method [18], and exponential rational function method [19] to get solutions.

Feng presented an effective technique to obtain travelling wave solutions of NPDEs, known as the FIM method [2022]. FIM is based on the ring theory and commutative algebra. FIM provides first integral of explicit form having polynomials as coefficients by applying the division theorem. Contrary to other methods, the benefits of FIM are to produce exact and explicit solutions without complicated and lengthy calculations [23-25]. Despite several advantages, FIM can only be applied to integrable PDEs.

The focus of this paper is to find the exact solutions of conformable biological models. It includes KPP and its 
derived models, namely, FHN and NW. KPP is a general form of equation and we can obtain different equations from KPP, for example, FHN, NW, and Cahn Allen. The considered models are significant in biology. KPP describes the genetic model for spread of dominant gene through population. The graphical solutions of the KPP equation can be used for diallel analysis as diallel analysis requires graphical solutions of genes. In diallel analysis, graphical representation of genes is required and some further calculations enable researchers to have point estimation of recessive genes and dominant genes instead of providing an interval of estimation [26]. The FHN equation is used in the study of intercellular trigger waves. Trigger waves are pulses and oscillatory waves; these waves switch from one stable steady state to another [27]. Similarly, the NW equation is applied for Faraday instability, chemical reactions, Rayleigh-Benard convection, and biological systems [28]. Various techniques have been established for solving the KPP equation, for instance, the discrimination algorithm [29], the homotopy perturbation technique [30], the differential transform method [31], the $\left(G^{\prime} / G\right)$-expansion method [32], and the homotopy analysis method [33]. Generally, the solutions of KPP equations are based on series solutions or numerical solutions. In this work, an effective technique named as FIM was adopted to acquire the exact solutions of KPP, FHN, and NW equations. The work is novel as the exact solutions of considered models using FIM are not presented before in the literature.

This paper consists of the following sections. Conformable derivative is described in Section 2; the proposed technique FIM is discussed in Section 3; the solutions of conformable KPP, FHN, and NW equations are presented in Section 4, and Section 5 contains summary and further recommendations.

\section{Preliminaries}

2.1. Derivative: Conformable. Conformable derivative is defined by Khalil et al. [34, 35].

Definition 1. The conformable derivative for function $h:[0, \infty) \rightarrow R$ of order $\beta$ is given as

$$
T_{\beta}(h)(t)=\lim _{\varepsilon \rightarrow 0} \frac{h\left(t+\varepsilon t^{1-\beta}\right)-h(t)}{\varepsilon},
$$

whereas $\beta \in(0,1)$ having $t>0$. If function $h$ is $\beta$-differentiable in $(0, p)$, here $p>0$ and $\lim _{t \rightarrow 0^{+}} h^{(\beta)}(t)$ exists, then at 0 , conformable derivative is represented as $h^{(\beta)}(0)=\lim _{t \rightarrow 0^{+}}$ $h^{(\beta)}(t)$.

The conformable integral for function $h$ is given as

$$
I_{\beta}^{p}(h)(t)=\int_{p}^{t} \frac{h(x)}{x^{1-\beta}} d x
$$

where $p \geq 0$ and $\beta \in(0,1]$.

Khalil et al. further proposed the succeeding theorem [34-36].
Theorem 2. Let the functions $v$ and $u$ at the point $t>0$ be $\beta$-differentiable; for $\beta \in(0,1]$ we have the following properties [34].

(1) $T_{\beta}(c u+d v)=c T_{\beta}(u)+d T_{\beta}(v) \forall c, d \in \mathbb{R}$

(2) $T_{\beta}\left(t^{r}\right)=r t^{r-\beta} \forall r \in \mathbb{R}$

(3) $T_{\beta}(D)=0 \forall v(t)=D$ (constant functions)

(4) $T_{\beta}(v u)=v T_{\beta}(u)+u T_{\beta}(v)$

(5) $T_{\beta}(u / v)=\left(v T_{\beta}(u)-u T_{\beta}(v)\right) / v^{2}$

(6) Furthermore, if we have a differentiable function $u$, then $T_{\beta}(u)(t)=t^{1-\beta}(d u / d t)$

The conformable derivative of any differential function at origin is zero; despite this flaw, several studies have been made on conformable derivative, as it explains higher order integration, sequential differentiation and integration, connection of differentiation and integration, property of linearity, derivative of constant function, quotient and product rule, chain rule, and power rule [34, 36-39]. Consequently, many researchers are working on the applicability of conformable derivative for real-world problems, such as Jacobi elliptical function expansion method used to solve conformable Boussinesq and combined Kdv-mKdv equation [40], conformable space-time fractional $(2+1)$ dimensional dispersive long wave equation [41], conformable heat equation [42], and conformable perturbed nonlinear Schrodinger equation [43].

\section{Methodology}

Here, the methodology of FIM is presented.

Step 1. Conformable PDE is given as follows:

$H\left(\frac{\partial^{\beta} u}{\partial t^{\beta}}, \frac{\partial^{\beta} u}{\partial z_{1}^{\beta}}, \frac{\partial^{\beta} u}{\partial z_{2}^{\beta}}, \cdots, \frac{\partial^{\beta} u}{\partial z_{r}^{\beta}}, \frac{\partial^{2 \beta} u}{\partial t^{2 \beta}}, \frac{\partial^{2 \beta} u}{\partial z_{1}^{\beta} \partial z_{1}^{\beta}}, \frac{\partial^{2 \beta} u}{\partial z_{2}^{\beta} \partial z_{2}^{\beta}} \cdots\right)=0$.

Step 2. Now using the following transformation

$$
u\left(z_{1}, z_{2}, \cdots, z_{r}, t\right)=U(Y) .
$$

Specifically in case of conformable derivative, the next transformation is applied as

$$
Y=\frac{m_{1} z_{1}^{\beta}+m_{2} z_{2}^{\beta}+\cdots+m_{r} z_{r}^{\beta} \pm p t^{\beta}}{\beta}
$$

The transformation defined in equation (5) will convert conformable PDE in nonlinear ODE.

$$
F\left(U(Y), U^{\prime}(Y), U^{\prime \prime}(Y), \cdots\right)=0
$$


where $U^{\prime}(Y)=d U(Y) / d Y$ and transformed variable is denoted by $Y$.

Step 3. We will take other independent variables as

$$
\begin{gathered}
U(Y)=Z(Y), \\
U_{Y}(Y)=Y(Y) .
\end{gathered}
$$

As a result, FIM will provide a system of ODEs (nonlinear) as

$$
\begin{aligned}
\frac{\partial Z}{\partial Y} & =Y(Y) \\
\frac{\partial Y}{\partial Y} & =G(Z(Y), Y(Y)) .
\end{aligned}
$$

Step 4. We attain general solutions after integrating equation (8). There is no precise or sound technique to obtain first integrals in case of plane independent (autonomous) system, so it is difficult to get even one first integral. To determine the first integral, the division theorem is utilized. Hence, a first integral is derived (cf. equation (8)) with the help of the division theorem. In this way, nonlinear ordinary differential equations (ODEs) can be reduced into a first-order ODE system (integrable) through the division theorem. Afterwards, solving the obtained system (cf. equation (8)), the exact solutions can be acquired.

The theorem for complex domain $\mathbb{C}$ and two variables is given as

Division Theorem. Consider polynomials $G(z, y)$ and $R(z, y)$, in complex domain $\mathbb{C}$, where $G(z y)$ is irreducible. If at all zero points of $R(z, y), G(z, y)$ vanishes, then another polynomial $H(z, y)$ exists in $\mathbb{C}(z, y)$ and the following equality holds:

$$
R(z, y)=G(z, y) H(z, y)
$$

\section{Implementation of FIM: Conformable KPP Equation and Its Derived Equations}

The exact solutions of KPP, FHN, and NW equations are presented in this section.

4.1. Conformable Space-Time Fractional KPP Equation. Andrey Kolmogorov, Ivan Petrovsky, and Nikolai Piskunov proposed a nonlinear PDE called the Kolmogorov Petrovsky Piskunov (KPP) equation to describe the genetic model for spread of dominant gene through population. Later, the KPP equation is applied in different natural sciences like in physics as combustion, in biology as propagation of nerve impulses, in chemical kinetics as propagation of concentration waves, and in plasma as evolution of set of duffing oscillators.
Consider conformable space-time fractional KPP equation defined as $[32,44]$

$$
\frac{\partial^{\beta} u}{\partial t^{\beta}}-\frac{\partial^{2 \beta} u}{\partial x^{2 \beta}}+\mu u+\eta u^{2}+\delta u^{3}=0, \quad x>0, t>0,
$$

where $\mu, \eta$, and $\delta$ are constants and $\beta \in(0,1)$.

First, we use conformable derivative with the following transformation:

$$
\begin{aligned}
Y & =\frac{m x^{\beta}}{\beta}+\frac{p t^{\beta}}{\beta}, \\
u(Y) & =u(x, t),
\end{aligned}
$$

where the transformation variable is $Y$. The transformation represented in equation (11) will provide the following conversions:

$$
\frac{\partial^{\beta}(.)}{\partial t^{\beta}}=p \frac{d(.)}{d Y}, \frac{\partial^{2 \beta}(.)}{\partial x^{2 \beta}}=m^{2} \frac{d^{2}(.)}{d Y^{2}} .
$$

Here, $m$ and $p$ are constants. Then, we get ODE by using equation (12) in equation (10):

$$
p \frac{d u}{d Y}-m^{2} \frac{d^{2} u}{d Y^{2}}+\mu u+\eta u^{2}+\delta u^{3}=0 .
$$

Now, we acquire a $2 \mathrm{D}$ system from equation (7) as

$$
\begin{aligned}
& \frac{d Z}{d Y}=Y, \\
& \frac{d Y}{d Y}=\frac{\mu}{m^{2}} Z+\frac{\eta}{m^{2}} Z^{2}+\frac{\delta}{m^{2}} Z^{3}+\frac{p}{m^{2}} Y .
\end{aligned}
$$

Afterwards, the division theorem will provide first integral. According to FIM, $Z$ and $Y$ are supposed to be nontrivial solutions of the system given (cf. equations (14) and (15)). Now, the division theorem provides us an irreducible polynomial $R(Z, Y)=\sum_{r=0}^{n} a_{r}(Z) Y^{r}$ in $\mathbb{C}[Z, Y]$ given as

$$
R(Z(Y), Y(Y))=\sum_{r=0}^{n} a_{r}(Z(Y)) Y(Y)^{r}=0
$$

where $a_{r}(Z) \neq 0$ and $r=0,1, \cdots, n$. Now, we have a polynomial of form $w(Z)+q(Z) Y$ in $\mathbb{C}[Z, Y]$ such that

$$
\frac{\partial R}{\partial Y}=\frac{\partial R}{\partial Z} \frac{\partial Z}{\partial Y}+\frac{\partial R}{\partial Y} \frac{\partial Y}{\partial Y}=(w(Z)+q(Z) Y)\left(\sum_{r=0}^{n} a_{r}(Z) Y^{r}\right)
$$

Using $n=1$ in equation (17) and equating coefficients for $Y^{r}(r=0,1)$, then we have following equations:

$$
\begin{aligned}
& a_{1}^{\prime}(Z)=a_{1}(Z) q(Z), \\
& a_{0}^{\prime}(Z)=w(Z) a_{1}(Z)+q(Z) a_{0}(Z)-a_{1}(Z) \frac{p}{m^{2}},
\end{aligned}
$$




$$
w(Z) a_{0}(Z)=a_{1}(Z) \frac{\mu}{m^{2}} Z+a_{1}(Z) \frac{\eta}{m^{2}} Z^{2}+a_{1}(Z) \frac{\delta}{m^{2}} Z^{3}
$$

Here, $a_{r}(Z)$ are polynomials in $Z$. As equation (18) shows $a_{1}(Z)$ has constant nature, hence $q(Z)=0$ and we can take $a_{1}(Z)=1$. We conclude that $\operatorname{deg}(w(Z))$ can only be 0 or 1 by using $a_{1}(Z)$ and $q(Z)$ in equations (19) and (20) and after balancing the functions $w(Z)$ and $a_{0}(Z)$ degrees. Now, we can take $w(Z)=A_{1} Z+A_{0}$; therefore, equation (19) takes the following form:

$$
a_{0}(Z)=\frac{1}{2} A_{1} Z^{2}+A_{0} Z-\frac{p}{m^{2}} Z+A_{2}
$$

where $A_{2}$ is an integrating constant.

Afterwards, the substitutions of values of $a_{0}(Z), w(Z)$ in equation (20) provide a system of nonlinear algebraic equations by equating the power of $Z$. Thus, as a result, we obtain various values of constants given as follows.

$$
\begin{aligned}
& A_{2}=0 \\
& A_{1}=\frac{\sqrt{2 \delta}}{m}, \\
& A_{0}=\frac{\eta}{m \sqrt{2 \delta}}+\frac{\sqrt{\eta^{2}-4 \mu \delta}}{m \sqrt{2 \delta}}, \\
& p=\frac{\eta m}{2 \sqrt{2 \delta}}+\frac{3 m}{2 \sqrt{2 \delta}} \sqrt{\eta^{2}-4 \mu \delta} .
\end{aligned}
$$

Case 1. The following constants are acquired as follows: we get

Substituting equations (21) and (22) into equation (16),

$$
Y(Y)=-\frac{1}{2} A_{1} Z^{2}-A_{0} Z+\frac{p}{m^{2}} Z
$$

Substitution of equation (23) into equation (14) provides the first solution of conformable fractional KPP equation.

$$
\begin{aligned}
& u_{1}(x, t) \\
& =-\frac{2 \mu \delta}{\delta \sqrt{\eta^{2}-4 \mu \delta}+\delta \eta-2 \gamma \mu \delta e^{(-\sqrt{2} / 4 m \sqrt{\delta})\left(\sqrt{\eta^{2}-4 \mu \delta}-\eta\right)\left(\left(m x^{\beta} / \beta\right)+\left(p t^{\beta} / \beta\right)\right)}} . \\
& A_{2}=0 \\
& A_{1}=\frac{\sqrt{2 \delta}}{m}, \\
& A_{0}=\frac{\eta}{m \sqrt{2 \delta}}-\frac{\sqrt{\eta^{2}-4 \mu \delta}}{m \sqrt{2 \delta}} \\
& p=\frac{\eta m}{2 \sqrt{2 \delta}}-\frac{3 m}{2 \sqrt{2 \delta}} \sqrt{\eta^{2}-4 \mu \delta} .
\end{aligned}
$$

Case 2. We get
Substituting equations (21) and (25) into equation (16), we obtain

$$
Y(Y)=-\frac{1}{2} A_{1} Z^{2}-A_{0} Z+\frac{p}{m^{2}} Z
$$

Substitution of equation (26) into equation (14) provides the second solution of conformable fractional KPP equation.

$$
\begin{aligned}
& u_{2}(x, t) \\
& =\frac{2 \mu \delta}{\delta \sqrt{\eta^{2}-4 \mu \delta}-\delta \eta+2 \gamma \mu \delta e^{(\sqrt{2} / 4 m \sqrt{\delta})\left(\sqrt{\eta^{2}-4 \mu \delta}+\eta\right)\left(\left(m x^{\beta} / \beta\right)+\left(p t^{\beta} / \beta\right)\right)}} . \\
& A_{2}=0, \\
& A_{1}=-\frac{\sqrt{2 \delta}}{m}, \\
& A_{0}=-\frac{\eta}{m \sqrt{2 \delta}}+\frac{\sqrt{\eta^{2}-4 \mu \delta}}{m \sqrt{2 \delta}}, \\
& p=-\frac{\eta m}{2 \sqrt{2 \delta}}+\frac{3 m \sqrt{\eta^{2}-4 \mu \delta}}{2 \sqrt{2 \delta}} .
\end{aligned}
$$

Case 3. We have

Substituting equations (21) and (28) into equation (16), we get

$$
Y(Y)=-\frac{1}{2} A_{1} Z^{2}-A_{0} Z+\frac{p}{m^{2}} Z
$$

Substitution of equation (29) into equation (14) provides the third solution of conformable fractional KPP equation.

$$
\begin{aligned}
& u_{3}(x, t) \\
& =\frac{2 \mu \delta}{\delta \sqrt{\eta^{2}-4 \mu \delta}-\delta \eta+2 \gamma \mu \delta e^{(-\sqrt{2} / 4 m \sqrt{\delta})\left(\sqrt{\eta^{2}-4 \mu \delta}+\eta\right)\left(\left(m x^{\beta} / \beta\right)+(p t \beta / \beta)\right)}} .
\end{aligned}
$$

$$
\begin{aligned}
& A_{2}=0 \\
& A_{1}=-\frac{\sqrt{2 \delta}}{m}, \\
& A_{0}=-\frac{\eta}{m \sqrt{2 \delta}}-\frac{\sqrt{\eta^{2}-4 \mu \delta}}{m \sqrt{2 \delta}}, \\
& p=-\frac{\eta m}{2 \sqrt{2 \delta}}-\frac{3 m \sqrt{\eta^{2}-4 \mu \delta}}{2 \sqrt{2 \delta}} .
\end{aligned}
$$

Case 4. We obtain

Substituting equations (21) and (31) into equation (16), we obtain

$$
Y(Y)=-\frac{1}{2} A_{1} Z^{2}-A_{0} Z+\frac{c}{m^{2}} Z
$$


Substitution of equation (32) into equation (14) provides the fourth solution of conformable fractional KPP equation.

$$
\begin{aligned}
& u_{4}(x, t) \\
& =-\frac{2 \mu \delta}{\delta \sqrt{\eta^{2}-4 \mu \delta}+\delta \eta-2 \gamma \mu \delta e^{(\sqrt{2} / 4 m \sqrt{\delta})\left(\sqrt{\eta^{2}-4 \mu \delta}-\eta\right)\left(\left(m x^{\beta} / \beta\right)+\left(p t^{\beta} / \beta\right)\right)}} .
\end{aligned}
$$

$$
\begin{aligned}
A_{0} & =0, \\
A_{1} & =\frac{\sqrt{2 \delta}}{m}, \\
A_{2} & =\frac{\mu}{m \sqrt{2 \delta}}, \\
p & =-\frac{\eta m}{\sqrt{2 \delta}} .
\end{aligned}
$$

Case 5. We get

Substituting equations (21) and (34) into equation (16), we obtain

$$
Y(Y)=-\frac{1}{2} A_{1} Z^{2}+\frac{p}{m^{2}} Z-A_{2}
$$

Substitution of equation (35) into equation (14) provides the fifth solution of conformable fractional KPP equation.

$$
\begin{aligned}
& u_{5}(x, t) \\
& =\frac{-\eta-\tan \left(\left(\left(\left(\left(m x^{\beta} / \beta\right)+\left(p t^{\beta} / \beta\right)\right)+\gamma\right) \sqrt{8 \mu \delta-2 \eta^{2}}\right) / 4 m \sqrt{\delta}\right) \sqrt{4 \mu \delta-\eta^{2}}}{2 \delta} .
\end{aligned}
$$

$$
\begin{aligned}
A_{0} & =0, \\
A_{1} & =-\frac{\sqrt{2 \delta}}{m}, \\
A_{2} & =-\frac{\mu}{m \sqrt{2 \delta}}, \\
p & =\frac{\eta m}{\sqrt{2 \delta}} .
\end{aligned}
$$

Case 6. We have

Substituting equations (21) and (37) into equation (16), we obtain

$$
Y(Y)=-\frac{1}{2} A_{1} Z^{2}+\frac{c}{m^{2}} Z-A_{2}
$$

Substitution of equation (38) into equation (14) provides the second solution of conformable fractional KPP equation.

$$
\begin{aligned}
& u_{6}(x, t) \\
& =\frac{-\eta+\tan \left(\left(\left(\left(\left(m x^{\beta} / \beta\right)+\left(p t^{\beta} / \beta\right)\right)+\gamma\right) \sqrt{8 \mu \delta-2 \eta^{2}}\right) / 4 m \sqrt{\delta}\right) \sqrt{4 \mu \delta-\eta^{2}}}{2 \delta} .
\end{aligned}
$$

The solutions $u_{1}, u_{2}, u_{3}, u_{4}, u_{5}, u_{6}$ are presented in Figure 1. For larger values of $\beta$, the solutions attain more height which is depicted in Figure 2. Figure 3 shows the graphical presentation in 2D plot of genes for the KPP equation. The graphical solutions of the KPP equation can be used in diallel analysis as observed in [26].

4.2. Conformable Time-Fractional FHN Equation. Richard Fitzhugh proposed a model for transmission of impulses in nerve axon in 1961. Nagumo et al. made the identical circuit in succeeding years and presented the model of an excitable system. FHN is derived from KPP on substituting $\mu=\xi, \eta=-(\xi+1)[45,46]$.

Consider conformable time-space fractional FHN equation as

$$
\frac{\partial^{\beta} u}{\partial t^{\beta}}-\frac{\partial^{2} u}{\partial x^{2}}-u(1-u)(u-\xi)=0,
$$

where $\beta \in(0,1)$ and $\xi \in(0,0.5]$.

First, we use the conformable derivative with the following transformation:

$$
\begin{gathered}
Y=x+\frac{p t^{\beta}}{\beta}, \\
u(Y)=u(x, t),
\end{gathered}
$$

where the transformation variable is $Y$. The transformation represented in equation (41) will provide the following conversions:

$$
\frac{\partial^{\beta}(.)}{\partial t^{\beta}}=p \frac{d(.)}{d Y}, \frac{\partial^{2}(.)}{\partial x^{2}}=\frac{d^{2}(.)}{d Y^{2}} .
$$

Here, $p$ is a constant. Then, we get an ODE by using equation (42) in equation (40):

$$
p \frac{d u}{d Y}-\frac{d^{2} u}{d Y^{2}}-u(1-u)(u-\xi)=0
$$

Now, we obtain a $2 \mathrm{D}$ system from equation (7) as

$$
\begin{aligned}
& \frac{d Z}{d Y}=Y, \\
& \frac{d Y}{d Y}=Z^{3}-Z^{2}+\xi Z-\xi Z^{2}+p Y .
\end{aligned}
$$

Afterwards, the division theorem will give first integrals. According to FIM, $Z$ and $Y$ are supposed to be nontrivial solutions of the system given (cf. equations (44) and (45)). Now, the division theorem provides us an irreducible polynomial $R(Z, Y)=\sum_{r=0}^{n} a_{r}(Z) Y^{r}$ in $\mathbb{C}[Z, Y]$ as

$$
R(Z(Y), Y(Y))=\sum_{r=0}^{n} a_{r}(Z(Y)) Y(Y)^{r}=0
$$



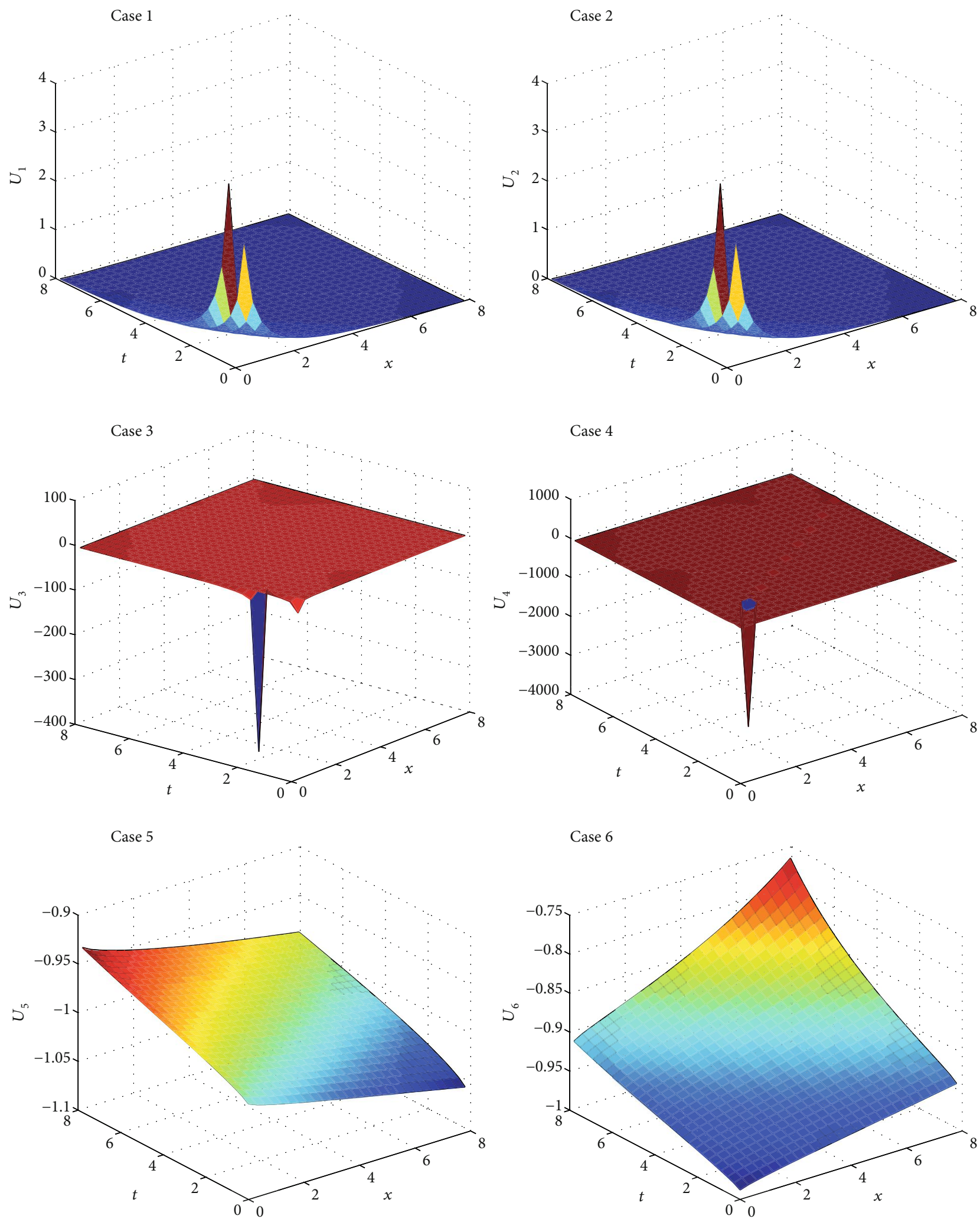

Figure 1: Solutions of conformable fractional KPP equation Cases $1-6$ using $\eta=1.93-2.25, m=1, \delta=1, \gamma=1$, and $\beta=0.8$. 


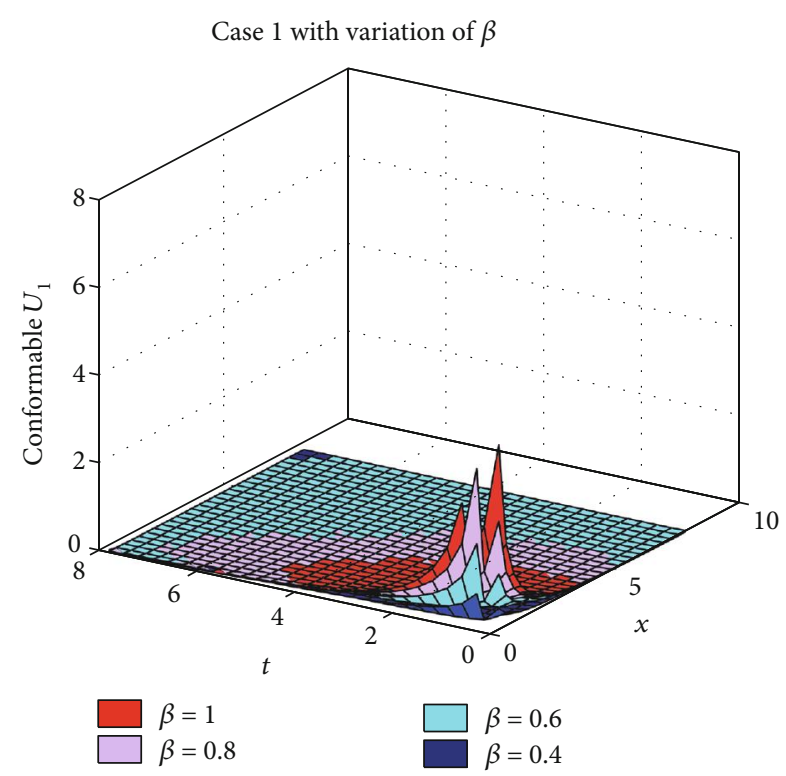

FIgURE 2: Solutions of conformable fractional KPP equation (Case 1) considering $\eta=2.1, m=1, \delta=1, \mu=1, \gamma=1, \beta=1, \beta=0.8, \beta=0.6$, and $\beta=0.4$.

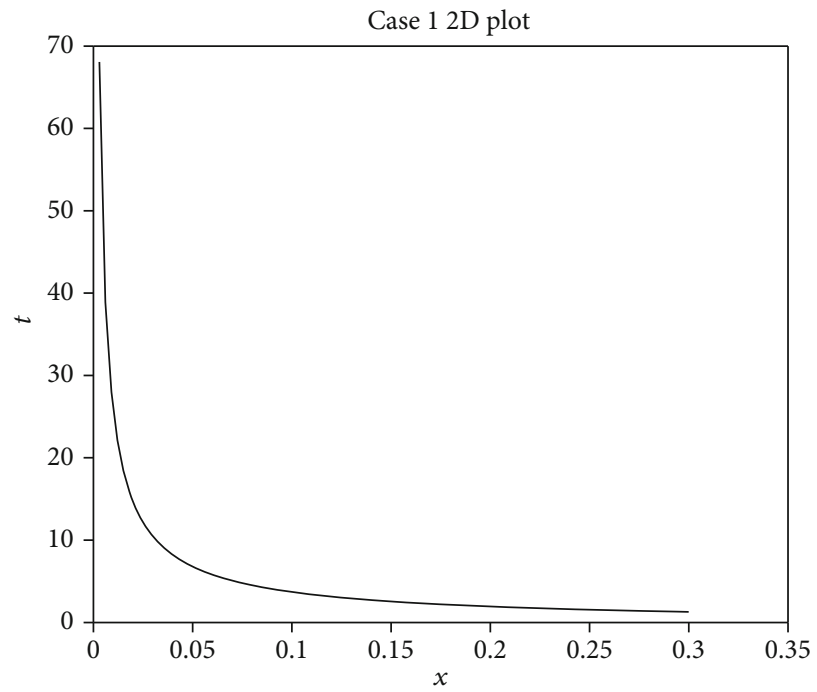

FIgURE 3: 2D plot of conformable fractional KPP equation (Case 1) using $\eta=2.1, m=1, \delta=1, \mu=1, \gamma=1$, and $\beta=0.8$.

where $a_{r}(Z) \neq 0$ and $r=0,1, \cdots, n$. Now, we have a polynomial of form $w(Z)+q(Z) Y$ in $\mathbb{C}[Z, Y]$ such that

$$
\frac{\partial R}{\partial Y}=\frac{\partial R}{\partial Z} \frac{\partial Z}{\partial Y}+\frac{\partial R}{\partial Y} \frac{\partial Y}{\partial Y}=(w(Z)+q(Z) Y)\left(\sum_{r=0}^{n} a_{r}(Z) Y^{r}\right) .
$$

Using $n=1$ in equation (47) and equating coefficients of $Y^{r}(r=0,1)$, then we have the following equations:

$$
a_{1}^{\prime}(Z)=a_{1}(Z) q(Z)
$$

$$
\begin{aligned}
a_{0}^{\prime}(Z) & =w(X) a_{1}(Z)+q(Z) a_{0}(Z)-p a_{1}(Z), \\
w(Z) a_{0}(Z) & =-a_{1}(Z) Z^{2}+\xi a_{1}(Z) Z+a_{1}(Z) Z^{3}-\xi a_{1}(Z) Z^{2} .
\end{aligned}
$$

Here, $a_{r}(Z)$ are polynomials in $Z$. As equation (48) shows $a_{1}(Z)$ has constant nature, thus $q(Z)=0$ and we can take $a_{1}$ $(Z)=1$. We conclude that $\operatorname{deg}(w(Z))$ can only be 0 or 1 by using $a_{1}(Z)$ and $q(Z)$ in equations (48) and (49) and after balancing the functions $w(Z)$ and $a_{0}(Z)$ degrees. Now, we can take $w(Z)=A_{1} Z+A_{0}$; therefore, equation (49) takes the following form:

$$
a_{0}(Z)=\frac{1}{2} A_{1} Z^{2}-A_{0}(Z)-p Z+A_{2}
$$

where $A_{2}$ is an integrating constant.

Afterwards, the substitutions of the values of $a_{0}(Z), w(Z)$ in equation (50) provide a system of nonlinear algebraic equations by equating the power of $Z$. Now, as a result, we have various constants given as follows.

$$
\begin{aligned}
A_{1} & =\sqrt{2}, \\
A_{0} & =0, \\
A_{2} & =\frac{\mu}{\sqrt{2}}, \\
p & =\frac{1+\xi}{\sqrt{2}} .
\end{aligned}
$$

Case 7. We get

Substituting equations (51) and (52) into equation (46), we get the following equation.

$$
Y(Y)=-\frac{\sqrt{2}}{2} Z^{2}+\frac{1+\xi}{\sqrt{2}} Z-\frac{\xi}{\sqrt{2}} .
$$

Substitution of equation (53) into equation (44) provides the first solution of conformable fractional FHN equation.

$$
\begin{aligned}
& u_{7}(x, t)
\end{aligned}
$$

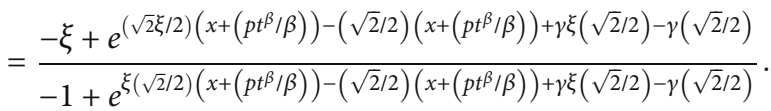

$$
\begin{aligned}
& A_{1}=-\sqrt{2}, \\
& A_{0}=0 \text {, } \\
& A_{2}=\frac{-\xi}{\sqrt{2}}, \\
& p=\frac{-(1+\xi)}{\sqrt{2}} \text {. }
\end{aligned}
$$

Case 8. We get 
Substituting equations (51) and (55) into equation (46), we get the subsequent equation.

$$
Y(Y)=\frac{\sqrt{2}}{2} Z^{2}-\frac{1+\xi}{\sqrt{2}} Z+\frac{\xi}{\sqrt{2}} .
$$

Substitution of equation (56) into equation (44) provides the second solution of conformable fractional FHN equation.

$$
\begin{aligned}
& u_{8}(x, t) \\
& =\frac{-\xi+e^{(\sqrt{2} \xi / 2)\left(x+\left(p t^{\beta} / \beta\right)\right)-(\sqrt{2} / 2)\left(x+\left(p t^{\beta} / \beta\right)\right)+\gamma \xi(\sqrt{2} / 2)-\gamma(\sqrt{2} / 2)}}{-1+e^{\xi(\sqrt{2} / 2)\left(x+\left(p t^{\beta} / \beta\right)\right)-(\sqrt{2} / 2)\left(x+\left(p t^{\beta} / \beta\right)\right)+\gamma \xi(\sqrt{2} / 2)-\gamma(\sqrt{2} / 2)}} .
\end{aligned}
$$

$$
\begin{aligned}
A_{1} & =\sqrt{2}, \\
A_{2} & =0, \\
A_{0} & =-\sqrt{2} \xi \\
p & =\frac{1}{\sqrt{2}}-\xi \sqrt{2} .
\end{aligned}
$$

Case 9. We have

Substituting equations (51) and (58) into equation (46), we obtain the following equation.

$$
Y(Y)=-\frac{\sqrt{2}}{2} Z^{2}+\frac{1}{\sqrt{2}} Z
$$

Substitution of equation (59) into equation (44) provides the third solution of conformable fractional FHN equation.

$$
\begin{aligned}
u_{9}(x, t) & =\frac{1}{1+\gamma e^{-(\sqrt{2} / 2)(x+(p t \beta / \beta))} .} \\
A_{1} & =\sqrt{2} \\
A_{2} & =0 \\
A_{0} & =-\sqrt{2} \\
p & =-\sqrt{2}+\xi \frac{1}{\sqrt{2}} .
\end{aligned}
$$

Case 10. We obtain

Substituting equations (51) and (61) into equation (46), we get

$$
Y(Y)=-\frac{\sqrt{2}}{2} Z^{2}+\frac{\xi}{\sqrt{2}} Z
$$

Substitution of equation (62) into equation (44) provides the fourth solution of conformable fractional FHN equation.

$$
\begin{aligned}
u_{10}(x, t) & =\frac{\xi}{1+\gamma \xi e^{-(\xi \sqrt{2} / 2)\left(x+\left(p t^{\beta} / \beta\right)\right)} .} \\
A_{1} & =-\sqrt{2}, \\
A_{2} & =0 \\
A_{0} & =\sqrt{2} \xi \\
p & =-\frac{1}{\sqrt{2}}+\xi \sqrt{2} .
\end{aligned}
$$

Case 11. We get

Substituting equations (51) and (64) into equation (46), we obtain

$$
Y(Y)=\frac{\sqrt{2}}{2} Z^{2}-\frac{1}{\sqrt{2}} Z .
$$

Substitution of equation (65) into equation (44) provides the fifth solution of conformable fractional FHN equation.

$$
u_{11}(x, t)=\frac{1}{1+\gamma e^{(\sqrt{2} / 2)\left(x+\left(p t^{\beta / \beta}\right)\right)}} .
$$

$$
\begin{gathered}
A_{1}=-\sqrt{2}, \\
A_{2}=0, \\
A_{0}=\sqrt{2}, \\
p=-\frac{\xi}{\sqrt{2}}+\sqrt{2} .
\end{gathered}
$$

Case 12. We have

Substituting equations (51) and (67) into equation (46), we get

$$
Y(Y)=\frac{\sqrt{2}}{2} Z^{2}-\frac{\xi}{\sqrt{2}} Z .
$$

Substitution of equation (68) into equation (44) provides the sixth solution of conformable fractional FHN equation.

$$
u_{12}(x, t)=\frac{\xi}{1+\gamma \xi e^{(\xi \sqrt{2} / 2)\left(x+\left(p t^{\beta} / \beta\right)\right)}} .
$$

The solutions $u_{7}, u_{8}, u_{9}, u_{10}, u_{11}, u_{12}$ are presented in Figure 4. For smaller value of $\beta$, solutions attain more height which is depicted in Figure 5.

4.3. Conformable Space-Time Fractional NW Equation. NW has wide applications in mechanical and chemical engineering, ecology, and biology [34]. NW can be derived from KPP by substituting $\mu=-1, \eta=0$, and $\delta=1$. 

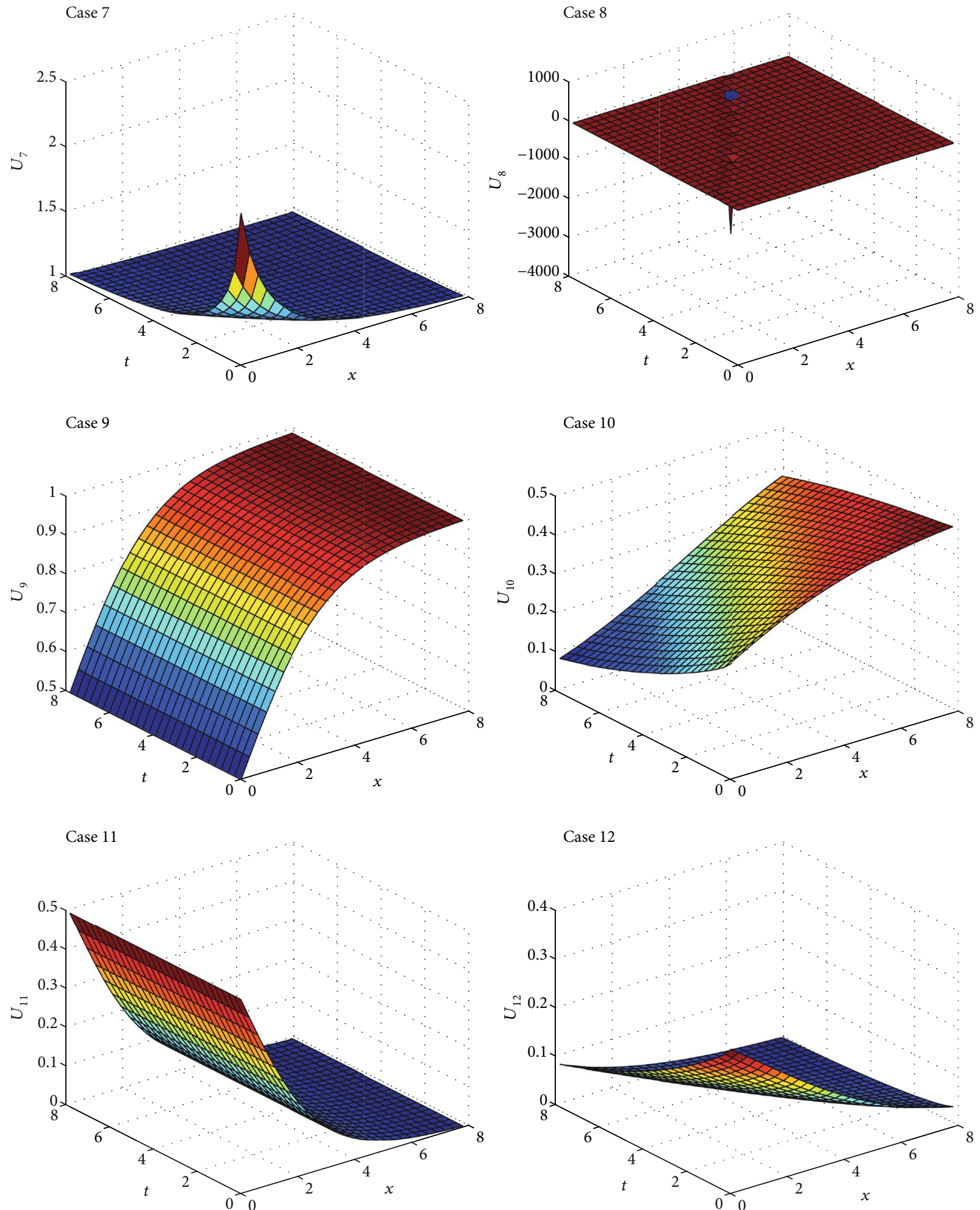

FIgURE 4: Conformable fractional FHN equation Cases 7-12 using $\beta=0.8, \gamma=1$, and $\xi=0.5$. 


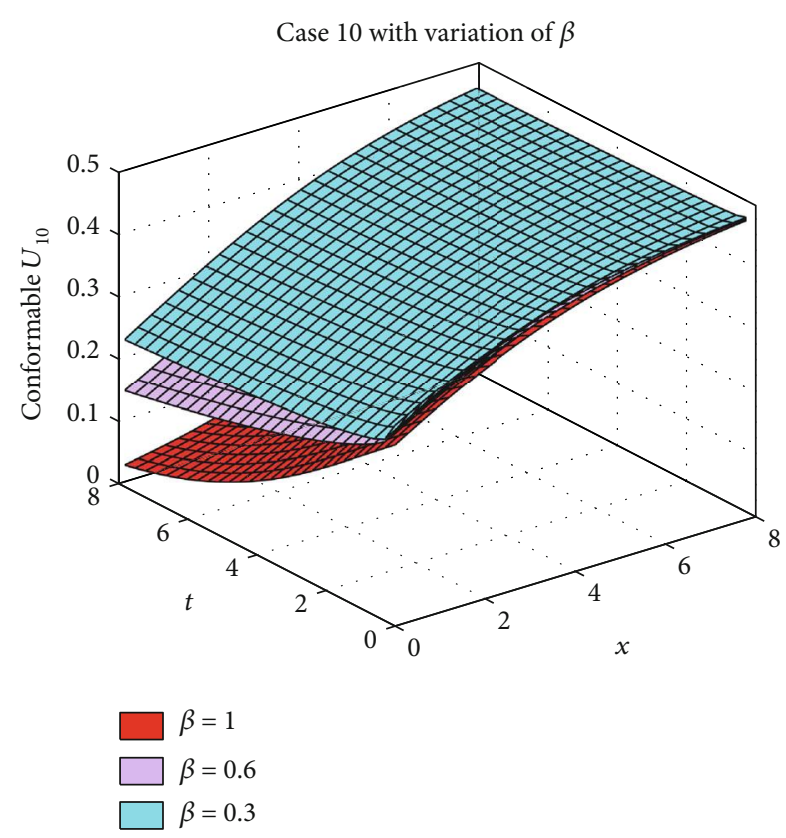

Figure 5: Conformable fractional FHN equation (Case 10) considering $\gamma=1, \xi=0.45, \beta=1, \beta=0.6$, and $\beta=0.3$. tion as

Consider conformable space-time fractional NW equa-

$$
\frac{\partial^{\beta} u}{\partial t^{\beta}}=K \frac{\partial^{\alpha} u}{\partial x^{\alpha}}+a u-b u^{q}
$$

where $\beta \in(0,1)$ and $\alpha \in(0,2], \beta>0, \alpha>1, a, b, K>0$ and $q$ is a positive integer. For $a=1, b=1, K=1$, and $\alpha=2$, equation (70) becomes

$$
\frac{\partial^{\beta} u}{\partial t^{\beta}}=\frac{\partial^{2} u}{\partial x^{2}}+u-u^{3}
$$

First, we use the conformable derivative with the following transformation:

$$
\begin{aligned}
Y & =x+\frac{p t^{\beta}}{\beta} \\
u(Y) & =u(x, t),
\end{aligned}
$$

where the transformation variable is $Y$. The transformation represented in equation (72) will provide the following conversions.

$$
\frac{\partial^{\beta}(\cdot)}{\partial t^{\beta}}=p \frac{d(.)}{d Y}, \frac{\partial^{2}(\cdot)}{\partial x^{2}}=\frac{d^{2}(\cdot)}{d Y^{2}},
$$

where $p$ is a constant. Then, we get ODE by using equation (73) in equation (71).

$$
p \frac{d u}{d Y}-\frac{d^{2} u}{d Y^{2}}-u+u^{3}=0
$$

Thus, we obtain a $2 \mathrm{D}$ system from equation (7) as

$$
\begin{aligned}
& \frac{d Z}{d Y}=Y, \\
& \frac{d Y}{d Y}=Z^{3}-Z+p Y .
\end{aligned}
$$

Afterwards, the division theorem will give first integrals. According to FIM, $Z$ and $Y$ are supposed to be nontrivial solutions of the system given (cf. equations (75) and (76)). Hence, the division theorem provides us irreducible polynomial $R(Z, Y)=\sum_{r=0}^{n} a_{r}(Z) Y^{r}$ in $\mathbb{C}[Z, Y]$ given as

$$
R(Z(Y), Y(Y))=\sum_{r=0}^{n} a_{r}(Z(Y)) Y(Y)^{r}=0
$$

where $a_{r}(Z) \neq 0$ and $r=0,1, \cdots, n$. Now, we have a polynomial of form $w(Z)+q(Z) Y$ in $\mathbb{C}[Z, Y]$ such that

$$
\frac{\partial R}{\partial Y}=\frac{\partial R}{\partial Z} \frac{\partial Z}{\partial Y}+\frac{\partial R}{\partial Y} \frac{\partial Y}{\partial Y}=(w(Z)+q(Z) Y)\left(\sum_{r=0}^{n} a_{r}(Z) Y^{r}\right)
$$

Using $n=1$ in equation (78) and equating coefficients of $Y^{r}(r=0,1)$, then we have following equations:

$$
\begin{aligned}
a_{1}^{\prime}(Z) & =a_{1}(Z) q(Z), \\
a_{0}^{\prime}(Z) & =w(Z)-p, \\
w(Z) a_{0}(Z) & =a_{1}(Z) Z^{3}-a_{1}(Z) Z .
\end{aligned}
$$

Here, $a_{r}(Z)$ are polynomials in $Z$. As equation (79) shows $a_{1}(Z)$ has constant nature, thus $q(Z)=0$ and we can take $a_{1}$ $(Z)=1$. We conclude that $\operatorname{deg}(w(Z))$ can only be 0 or 1 by using $a_{1}(Z)$ and $q(Z)$ in equations (80) and (81) and after balancing the functions $w(Z)$ and $a_{0}(Z)$ degrees. Now, we can take $w(Z)=A_{1} Z+A_{0}$; therefore, equation (80) takes the following form:

$$
a_{0}(Z)=\frac{1}{2} A_{1} Z^{2}+A_{0} Z-p Z+A_{2}
$$

where $A_{2}$ is an integrating constant.

Afterwards, the substitutions of the values of $a_{0}(Z), w(Z)$ in equation (81) provide a system of nonlinear algebraic equations by equating coefficients of power of $Z$. Thus, we have various constants as given below.

$$
\begin{aligned}
& A_{1}=\sqrt{2}, \\
& A_{0}=0, \\
& A_{2}=-\frac{1}{\sqrt{2}}, \\
& p=0 .
\end{aligned}
$$



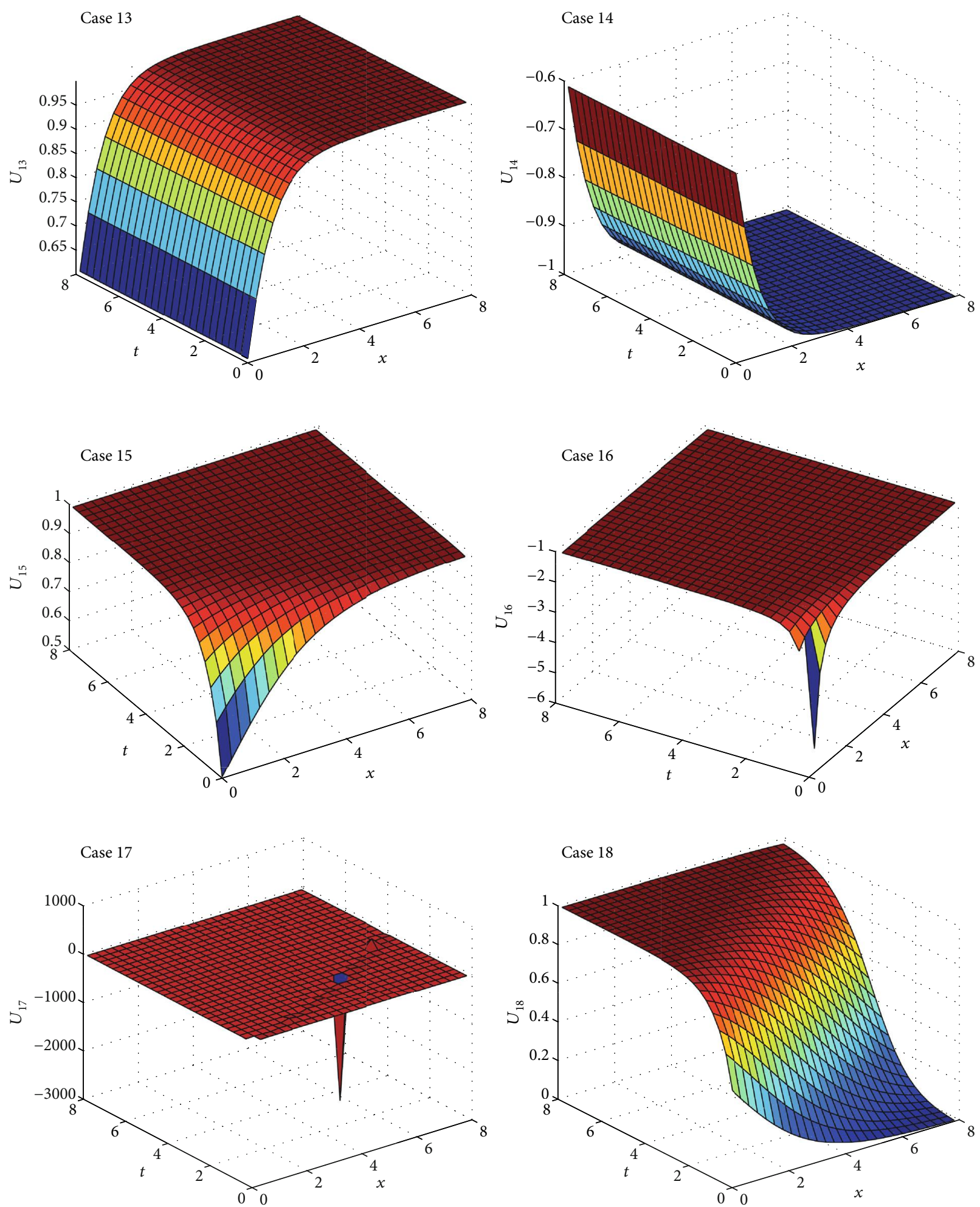

Figure 6: Conformable fractional NW equation Cases $13-18$ using $\beta=0.8$ and $\gamma=1$.

Substituting equations (82) and (83) into equation (77), we get

$$
Y(Y)=-\frac{\sqrt{2}}{2} Z^{2}+\frac{1}{\sqrt{2}} .
$$

Substitution of equation (84) into equation (75) provides the first solution of conformable fractional NW equation.

$$
u_{13}(x, t)=\tanh \left(\frac{\sqrt{2}}{2}\left(x+\frac{p t^{\beta}}{\beta}\right)+\frac{\gamma \sqrt{2}}{2}\right) .
$$




$$
\begin{aligned}
A_{1} & =-\sqrt{2}, \\
A_{0} & =0, \\
A_{2} & =\frac{1}{\sqrt{2}}, \\
p & =0 .
\end{aligned}
$$

\section{Case 14. We get}

Substituting equations (82) and (86) into equation (77), we obtain

$$
Y(Y)=\frac{\sqrt{2}}{2} Z^{2}-\frac{1}{\sqrt{2}} .
$$

Substitution of equation (87) into equation (75) provides the second solution of conformable fractional NW equation.

$$
\begin{gathered}
u_{14}(x, t)=-\tanh \left(\frac{\sqrt{2}}{2}\left(x+\frac{p t^{\beta}}{\beta}\right)+\frac{\gamma \sqrt{2}}{2}\right) . \\
A_{1}=\sqrt{2} \\
A_{2}=0 \\
A_{0}=\sqrt{2} \\
p=\frac{3}{\sqrt{2}} .
\end{gathered}
$$

\section{Case 15. We have}

Substituting equations (82) and (89) into equation (77), we get

$$
Y(Y)=-\frac{\sqrt{2}}{2} Z^{2}+\frac{1}{\sqrt{2}} Z .
$$

Substitution of equation (90) into equation (75) provides the third solution of conformable fractional NW equation.

$$
\begin{gathered}
u_{15}(x, t)=\frac{1}{1+\gamma e^{-(\sqrt{2} / 2)\left(x+\left(p t^{\beta} / \beta\right)\right)}} . \\
A_{1}=-\sqrt{2} \\
A_{2}=0 \\
A_{0}=\sqrt{2} \\
p=\frac{3}{\sqrt{2}} .
\end{gathered}
$$

Case 16. We obtain

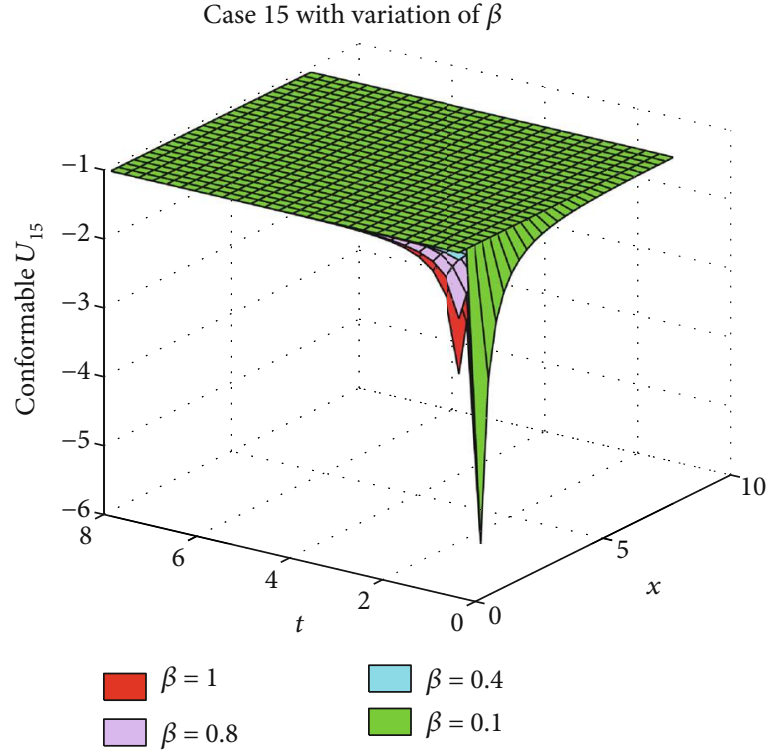

Figure 7: Conformable fractional NW equation (Case 15) considering $\gamma=1, \beta=1, \beta=0.8, \beta=0.4$, and $\beta=0.1$.

Substituting equations (82) and (92) into equation (77), we get

$$
Y(Y)=\frac{\sqrt{2}}{2} Z^{2}+\frac{1}{\sqrt{2}} Z .
$$

Substitution of equation (93) into equation (75) provides the fourth solution of conformable fractional NW equation.

$$
u_{16}(x, t)=\frac{1}{-1+\gamma e^{-(\sqrt{2} / 2)\left(x+\left(p t^{\beta} / \beta\right)\right)}} .
$$

$$
\begin{aligned}
A_{1} & =\sqrt{2}, \\
A_{2} & =0, \\
A_{0} & =-\sqrt{2}, \\
p & =-\frac{3}{\sqrt{2}} .
\end{aligned}
$$

Case 17. We get

Substituting equations (82) and (95) into equation (77), we obtain

$$
Y(Y)=-\frac{\sqrt{2}}{2} Z^{2}-\frac{1}{\sqrt{2}} Z .
$$

Substitution of equation (96) into equation (75) provides the fifth solution of conformable fractional NW equation.

$$
u_{17}(x, t)=\frac{1}{-1+\gamma e^{(\sqrt{2} / 2)\left(x+\left(p t^{\beta} / \beta\right)\right)}} .
$$




$$
\begin{aligned}
A_{1} & =-\sqrt{2}, \\
A_{2} & =0, \\
A_{0} & =-\sqrt{2}, \\
p & =-\frac{3}{\sqrt{2}} .
\end{aligned}
$$

Case 18. We have

Substituting equations (82) and (98) into equation (77), we obtain

$$
Y(Y)=\frac{\sqrt{2}}{2} Z^{2}-\frac{1}{\sqrt{2}} Z .
$$

Substitution of equation (99) into equation (75) provides the sixth solution of conformable fractional NW equation.

$$
u_{18}(x, t)=\frac{1}{1+\gamma e^{(\sqrt{2} / 2)\left(x+\left(p t^{\beta} / \beta\right)\right)}} .
$$

The solutions $u_{13}, u_{14}, u_{15}, u_{16}, u_{17}, u_{18}$ are presented in Figure 6 . In Figure 7, the results reveal that the peaks are getting sharper and sharper by reducing the value of $\beta$.

\section{Conclusion}

The purpose of this paper was to find new exact solutions of some conformable biological models. The considered models were KPP, FHN, and NW. FIM was employed to obtain the solutions of fractional KPP, FHN, and NW equations. The proposed method was found brief and direct. The results indicate that FIM is one of the best techniques to calculate exact solutions of nonlinear fractional order problems appearing in biology, physics, and engineering.

\section{Data Availability}

The data used to support the findings of this study are included within the article.

\section{Conflicts of Interest}

The authors declare that they have no conflicts of interest.

\section{Authors' Contributions}

Shumaila Javeed and Dumitru Baleanu were responsible for the conceptualization. Shumaila Javeed, Sidra Riaz, and Hadi Rezazadeh were responsible for the formal analysis. Shumaila Javeed and Sidra Riaz were responsible for the investigation. Shumaila Javeed and Sidra Riaz were responsible for the methodology. Dumitru Baleanu, Yu-Ming Chu, and Hadi Rezazadeh were responsible for the project administration. Dumitru Baleanu and Yu-Ming Chu were responsible for the resources. Hadi Rezazadeh was responsible for the software. Shumaila Javeed, Dumitru Baleanu, and Yu-Ming Chu were responsible for the supervision. Sidra Riaz, Yu-
Ming Chu, and Hadi Rezazadeh were responsible for the validation. Shumaila Javeed and Sidra Riaz were responsible for writing the original draft. Shumaila Javeed and Yu-Ming Chu were responsible for writing, reviewing, and editing.

\section{Acknowledgments}

The research was supported by the National Natural Science Foundation of China (Grant Nos. 11971142, 11871202, 61673169, 11701176, 11626101, and 11601485).

\section{References}

[1] P. J. Olver, Introduction to partial differential equations, Springer, New York, 2013.

[2] W. Gao, H. Rezazadeh, Z. Pinar, H. M. Baskonus, S. Sarwar, and G. Yel, "Novel explicit solutions for the nonlinear Zoomeron equation by using newly extended direct algebraic technique," Optical and Quantum Electronics, vol. 52, no. 1, pp. 1-13, 2020.

[3] H. Rezazadeh, A. Korkmaz, M. Eslami, and S. M. MirhosseiniAlizamini, "A large family of optical solutions to KunduEckhaus model by a new auxiliary equation method," Optical and Quantum Electronics, vol. 51, no. 3, 2019.

[4] J. G. Liu, M. Eslami, H. Rezazadeh, and M. Mirzazadeh, "Rational solutions and lump solutions to a non-isospectral and generalized variable-coefficient Kadomtsev-Petviashvili equation," Nonlinear Dynamics, vol. 95, no. 2, pp. 10271033, 2019.

[5] A. A. Kilbas, H. M. Shrivastava, and J. J. Trujillo, "Theory and applications of fractional differential equations," in North-Holland Mathematics Studies, vol. 204, pp. 15-23, Elsevier Science, Amsterdam, the Netherlands, 2006.

[6] K. S. Miller and B. Ross, An Introduction to the Fractional Calculus and Fractional Differential Equations, Wiley, New York, USA, 1993.

[7] I. Podlubny, Fractional differential equations: an introduction to fractional derivatives, fractional differential equations, to methods of their solution and some of their applications, vol. 198, Academic press, New York, 1998.

[8] D. Elizarraraz and L. Verde-Star, "Fractional divided differences and the Solution of differential equations of fractional order," Advances in applied mathematics, vol. 24, no. 3, pp. 260-283, 2000.

[9] L. Levi and F. Payroutet, "A time-fractional step method for conservation lawrelated obstacle problems," Advances in applied mathematics, vol. 27, no. 4, pp. 768-789, 2001.

[10] J. H. He, "Fractal calculus and its geometrical explanation," Results in Physics, vol. 10, pp. 272-276, 2018.

[11] X.-J. Yang, J. A. Tenreiro Machado, and D. Baleanu, "Exact TRAVELING-WAVE solution for local fractional Boussinesq equation in fractal domain," Fractals, vol. 25, no. 4, article 1740006, 2017.

[12] Y. Xiao-Jun, G. Feng, and H. M. Srivastava, "Non-differentiable exact solutions for the non-linear odes defined on fractal sets," Fractals, vol. 25, no. 4, 2017.

[13] M. Dehghan, J. Manafian, and A. Saadatmandi, "Solving nonlinear fractional partial differential equations using the homotopy analysis method," Numerical Methods for Partial Differential Equations, vol. 26, no. 2, pp. 448-479, 2010. 
[14] S. Momani and Z. Odibat, "Homotopy perturbation method for nonlinear partial differential equations of fractional order," Physics Letters A, vol. 365, no. 5-6, pp. 345-350, 2007.

[15] A. S. Elwakil and M. A. Abdou, "New exact travelling wave solutions using modified extended tanh-function method," Chaos, Solitons and Fractals, vol. 31, no. 4, pp. 840-852, 2007.

[16] E. Fan, "Extended tanh-function method and its applications to nonlinear equations," Physics Letters A, vol. 277, no. 4-5, pp. 212-218, 2000.

[17] T. C. Xia and L. Wang, "New explicit and exact solutions for the Nizhnik-Novikov-Vesselov equation," Applied Mathematics E-Notes, vol. 1, pp. 139-142, 2001.

[18] S. Zhang and H. Q. Zhang, "Fractional sub-equation method and its applications to nonlinear fractional PDEs," Physics Letters A, vol. 375, no. 7, pp. 1069-1073, 2011.

[19] E. Aksoy, M. Kaplan, and A. Bekir, "Exponential rational function method for space-time fractional differential equations," Waves in Random and Complex Media, vol. 26, no. 2, pp. 142-151, 2015.

[20] Z. Feng, "On explicit exact solutions to the compound Burgers-KdV equation,” Physics Letters A, vol. 293, no. 1-2, pp. 57-66, 2002.

[21] M. Eslami and H. Rezazadeh, "The first integral method for Wu-Zhang system with conformable time-fractional derivative," Calcolo, vol. 53, no. 3, pp. 475-485, 2016.

[22] H. Rezazadeh, J. Manafian, F. S. Khodadad, and F. Nazari, "Traveling wave solutions for density-dependent conformable fractional diffusion-reaction equation by the first integral method and the improved \$\$ $\$ \operatorname{textbf}\{\tan \} \backslash \operatorname{left}(\{\{\backslash$ mathbf $\{\backslash-$ $\operatorname{frac}\{1\}\{2\}\}\}\{\backslash$ boldsymbol $\{\backslash$ varphi $\}\} \backslash \operatorname{left}(\{\backslash$ boldsymbol $\{\backslash$ upxi $\}\}$ \right)\} \right)\$\$ tan $12 \varphi \xi$-expansion method," Optical and Quantum Electronics, vol. 50, no. 3, 2018.

[23] S. Javeed, S. Saif, A. Waheed, and D. Baleanu, "Exact solutions of fractional $\mathrm{mBBM}$ equation and coupled system of fractional Boussinesq-Burgers," Results in physics, vol. 9, pp. 1275-1281, 2018.

[24] S. Javeed, S. Saif, and D. Baleanu, "New exact solutions of fractional Cahn-Allen equation and fractional DSW system," Advances in difference equations, vol. 2018, no. 1, 2018.

[25] S. Javeed, S. Riaz, K. S. Alimgeer, M. Atif, A. Hanif, and D. Baleanu, "First integral technique for finding exact solutions of higher dimensional mathematical physics models," Symmetry, vol. 11, no. 6, p. 783, 2019.

[26] E. Efe, "An alternative method in diallel analysis obtaining the values of two Vxi points where parabola $\mathrm{W} * \mathrm{r}=[\mathrm{VoloxVr}] 1 / 2$ is cut by regression line $\mathrm{W}^{\prime}=\mathrm{a}^{\prime}+\mathrm{V}_{\mathrm{r}}$ and dividing the distance between those points into four equal parts," 3rd Balkan Conference on Operational research, pp. 16-19, 1995.

[27] L. Gelens, G. A. Anderson, E. James, and J. Ferrell, Spatial trigger waves: positive feedback gets you a long way, 2014.

[28] M. M. A. Mahgoub and A. K. H. Sedeeg, "On the solution of Newell-Whitehead-Segel equation," American Journal of Mathematical and computer modelling, vol. 1, no. 1, pp. 21-24, 2016.

[29] D. S. Wang and H. B. Li, "Single and multi-solitary wave solutions to a class of nonlinear evolution equations," Journal of Mathematical Analysis and Applications, vol. 343, no. 1, pp. 273-298, 2008.

[30] K. A. Gepreel, “The homotopy perturbation method applied to the nonlinear fractional Kolmogorov "Petrovskii" Piskunov equations," Applied Mathematics Letters, vol. 24, no. 8, pp. 1428-1434, 2011.
[31] L. N. Song and W. G. Wang, "Approximate solutions of nonlinear fractional Kolmogorov-Petrovskii-Piskunov equations using an enhanced algorithm of the generalized twodimensional differential transform method," Communications in Theoretical Physics, vol. 58, no. 2, pp. 182-188, 2012.

[32] J. Feng, W. Li, and Q. Wan, "Using G'G-expansion method to seek the traveling wave solution of Kolmogorov-PetrovskiiPiskunov equation," Applied Mathematics and Computation, vol. 217, no. 12, pp. 5860-5865, 2011.

[33] G. Hariharan, "The homotopy analysis method applied to the Kolmogorov-Petrovskii-Piskunov (KPP) and fractional KPP equations," Journal of Mathematical Chemistry, vol. 51, no. 3, pp. 992-1000, 2013.

[34] R. Khalil, M. Al-Horani, A. Yousef, and M. Sababheh, "A new definition of fractional derivative," Journal of Computational and Applied Mathematics, vol. 264, pp. 65-70, 2014.

[35] D. Zhao and M. Luo, "General conformable fractional derivative and its physical interpretations," Calcoolo, vol. 54, no. 3, article 903917, 2017.

[36] A. Atangana, D. Baleanu, and A. Alsaedi, "New properties of conformable derivative," Open Mathematics, vol. 13, no. 1, 2015.

[37] J. H. He, S. K. Elegan, and Z. P. Li, "Geometrical explanation of the fractional complex transform and derivative chain rule for fractional calculus," Physics Letters A, vol. 376, no. 4, pp. 257259, 2012.

[38] T. Abdeljawad, "On conformable fractional calculus," Journal of Computational and Applied Mathematics, vol. 279, pp. 5766, 2015.

[39] N. Benkhettoua, S. Hassania, and D. F. M. Torres, "A conformable fractional calculus on arbitrary time scales," Journal of King Saud University - Science, vol. 28, no. 1, pp. 93-98, 2016.

[40] O. Tasbozan, Y. Cenesiz, and A. Kurt, "New solutions for conformable fractional Boussinesq and combined Kdv-mKdv equations using Jacobi elliptic function expansion method," The european Physical Journal Plus, vol. 131, no. 7, p. 244, 2016.

[41] M. Eslami, "Solutions for space time fractional $(2+1)$ dimensional dispersive long wave equations," Iranian Journal of science and technology, Transaction A: Science, vol. 41, 2017.

[42] M. A. Hammad and R. Khalil, "Conformable fractional heat equation," International Journal of Pure and Applied Mathematical Sciences, vol. 94, pp. 215-221, 2014.

[43] A. Neirameh, "New soliton solutions to the fractional perturbed nonlinear Schrodinger equation with power law nonlinearity," SeMA Journal, vol. 73, 2015.

[44] G. Gaeta, C. Reless, M. Peyard, and T. Dauxois, "Simple models of non-linearDNA dynamics," Rivista del Nuovo cimento, vol. 17, no. 4, pp. 1-48, 1994.

[45] S. Z. Rida, A. M. A. El-Sayed, and A. M. A. Arafa, "On the solutions of time-fractional reaction-diffusion equations," Communications in Nonlinear Science and Numerical Simulation, vol. 15, no. 12, pp. 3847-3854, 2010.

[46] N. A. Khan, N. Khan, A. Ara, and M. Jamil, "Approximate analytical solutions of fractional reaction-diffusion equations," Journal of King Saud University - Science, vol. 24, no. 2, pp. 111-118, 2012. 